\title{
Using differentiation matrices for pseudospectral method solve Duffing Oscillator
}

\author{
L. A. Nhat
}

PhD student of RUDN University, Moscow 117198, Russia.

And Lecture at Tan Trao University, Tuyen Quang province, Vietnam.

Communicated by R. Saadati

\begin{abstract}
This article presents an approximate numerical solution for nonlinear Duffing Oscillators by pseudospectral (PS) method to compare boundary conditions on the interval $[-1,1]$. In the PS method, we have been used differentiation matrix for Chebyshev points to calculate numerical results for nonlinear Duffing Oscillators. The results of the comparison show that this solution had the high degree of accuracy and very small errors. The software used for the calculations in this study was Mathematica V.10.4.
\end{abstract}

Keywords: Duffing oscillator, pseudospectral methods, differential matrix, Duffing system, Chebyshev points.

2010 MSC: 34B15, 41A50, 65L10.

(C)2018 All rights reserved.

\section{Introduction}

In science and engineering, the Duffing Oscillator was a common model for nonlinear phenomena. The most general forced form of the Duffing equation is:

$$
\frac{\partial^{2}}{\partial t^{2}} x(t)+\alpha \frac{\partial}{\partial t} x(t)+\beta x(t)^{3}+\gamma x(t)=\delta \cos (\theta t), \quad-1 \leqslant t \leqslant 1, \quad x(-1)=0, x(1)=0,
$$

where $\alpha, \beta, \gamma, \delta, \theta$ are parameters: $\alpha$ controls the amount of damping; $\beta$ controls the amount of nonlinearity in the restoring force; $\gamma$ controls the linear stiffness; $\delta$ is the amplitude of the periodic driving force; $\theta$ is the angular frequency of the periodic driving force.

Equation (1.1) depends on the different $\gamma, \beta$, we had some special cases: $\gamma>0, \beta>0$ : Hard Spring Duffing Oscillator; $\gamma>0, \beta<0$ : Soft Spring Duffing Oscillator; $\gamma<0, \beta>0$ : Inverted Duffing Oscillator;

\footnotetext{
${ }^{*}$ Corresponding author

Email address: leanhnhat@tuyenquang.edu.vn (L. A. Nhat)

doi: $10.22436 /$ jnsa.011.12.04
}

Received: 2018-06-17 Revised: 2018-08-05 Accepted: 2018-08-19 
$\gamma=0, \beta>0$ : Nonharmonic Duffing Oscillator. These special cases had been extensively studied in the literature [7].

Several approaches have been studied so far dealing with the nonlinear Duffing Oscillators such as The differential transform method [12]; The Jacobi elliptic function cn [16]; The analysis method [1, 6, 8]; The Taylor Expansion [5]; The Legendre pseudospectral method [14, 15]; A Chebyshev collocation algorithm [13]; The Enhanced Cubication Method [4]; The Improved Taylor Matrix Method [2]; The Postverification Method [10], the energy balance method [9].

\section{Pseudospectral method using differential matrix for Chebyshev points}

Let $p(x)$ a polynomial of degree $n$, and we know that it is valued at the points $p\left(x_{0}\right), p\left(x_{1}\right), \ldots, p\left(x_{n}\right)$, then the first and second derivatives $p(x)$ at the same points are expressed in matrix form:

$$
p^{\prime}\left(x_{j}\right)=D p\left(x_{j}\right), p^{\prime \prime}\left(x_{j}\right)=D^{2} p\left(x_{j}\right), \quad j=0,1, \ldots, n,
$$

where $\mathrm{D}=\left\{\mathrm{d}_{\mathrm{ij}}\right\}$ is the so-called differentiation matrix [11]. In case when the Chebyshev-Gauss-Lobatto points are chose as the collocation points $y_{k}=\cos (k \pi / n),[3]$

$$
D_{i, j}= \begin{cases}\frac{1+2 n^{2}}{6} & i=j=0 \\ \frac{c_{i}}{2 c_{j}} \sin [(i+j) \pi /(2 n)] \sin [(i-j) \pi /(2 n)] & i \neq j \\ \frac{\cos (j \pi / n)}{2 \sin (j \pi / n)} & 0<i=j<n \\ -\frac{1+2 n^{2}}{6} & i=j=n\end{cases}
$$

here $c_{k}=1$ when $k=1,2, \ldots, n-1$ and $c_{k}=2$ when $k=0, n$. The application of differential algebra in ordinary differential equations can also extend to nonlinear differential equations, so we transformed the matrix D into matrices [11]:

$$
\begin{array}{ll}
\mathrm{E}^{(1)}=\left\{\mathrm{d}_{\mathrm{ij}}\right\}, & 1 \leqslant \mathrm{i}, \mathrm{j} \leqslant \mathrm{n}-1 \\
e_{0}^{(1)}=\left\{\mathrm{d}_{\mathrm{i} 0}\right\}, e_{\mathrm{n}}^{(1)}=\left\{\mathrm{d}_{\mathrm{in}}\right\}, & 0<\mathrm{i}<\mathrm{n}
\end{array}
$$

for a first-order differential element, the form $u^{\prime}\left(x_{i}\right)=E^{(1)} u\left(x_{i}\right)$.

With a second-order differential element, we use $D^{2}=d_{i j}^{2}$ and define the matrices:

$$
\begin{array}{ll}
E^{(2)}=\left\{d_{i j}^{2}\right\}, & 1 \leqslant i, j \leqslant n-1 \\
e_{0}^{(2)}=\left\{d_{i 0}^{2}\right\}, e_{n}^{(2)}=\left\{d_{i n}^{2}\right\}, & 0<i<n
\end{array}
$$

has the form $u^{\prime \prime}\left(x_{i}\right)=E^{(2)} u\left(x_{i}\right)$.

\section{The approximation of the nonlinear Duffing Oscillators}

If $\alpha=0$, then (1.1) become the form:

$$
\frac{\partial^{2}}{\partial t^{2}} x(t)=f(x(t))
$$

with boundary conditions $x(-1)=a, x(1)=b$, then follow [11] we have

$$
E^{(2)} x\left(t_{j}\right)=f\left(x\left(t_{j}\right)\right), \quad j=\overline{1, n-1},
$$

here $f\left(x\left(t_{j}\right)\right)$ denotes the vector with elements $\left\{f\left(x_{n}\left(t_{j}\right)\right)\right\}$. To find the solution of the equation (3.1), we will be proceed with an iterative procedure with the forming equation:

$$
E^{(2)} x^{(k)}=f\left(x^{(k)}\right), \quad k=1,2, \ldots
$$

It is important to determine the iterative equation (3.3). The iterative procedure is simple, we assumed $u^{(0)}=$ const, then found $u^{(1)}, u^{(2)}, \ldots$, stop it until the error $\varepsilon=\left|u^{(k)}-u^{(k-1)}\right|<\varepsilon_{0}$. 
Example 3.1. We consider the following nonlinear oscillator:

$$
x^{\prime \prime}+x+\beta x^{3}=0, \quad-1 \leqslant t \leqslant 1, \quad x(-1)=a, x(1)=b .
$$

We will have an iterative procedure:

$$
\left(E^{(2)}+R\right) x^{(k)}=\beta\left(x^{(k-1)}\right)^{3}-b e_{0}^{(2)}-a e_{n}^{(2)}
$$

in which $\mathrm{R}$ is a square identity matrix.

In the case of the more general equation (1.1), we transformed into the form:

$$
E^{(2)} x\left(t_{j}\right)+\alpha E^{(1)} x\left(t_{j}\right)+\gamma x\left(t_{j}\right)=\delta \cos \left(\theta t_{j}\right)-\beta x^{(*)}\left(t_{j}\right)^{3} .
$$

Therefore it can then be written in iterative matrix notation as

$$
\left(\mathrm{E}^{(2)}+\mathrm{QE} \mathrm{E}^{(1)}+\mathrm{R}\right) \chi^{(\mathrm{k})}=\delta \cos \left(\theta \mathrm{t}_{j}\right)-\beta\left(x^{(\mathrm{k}-1)}\right)^{3}
$$

in where $Q, R$ is a square matrix with the elements $\alpha, \gamma$ on the main diagonal. Equation (3.7) is repeated until the error $\varepsilon<\varepsilon_{0}$.

\section{Results}

a. The Hard Spring Duffing Oscillator case

We gave the error $\varepsilon_{0}=10^{-8}$, the points $n=64$. And $\alpha=0.9, \beta=0.5, \gamma=0.7, \delta=0.65, \theta=$ 4. Table 1 shows a comparison of numerical results and error with Mathematica calculations for Hard Spring Duffing Oscillator case. In the Fig. 1, the results were calculated base on the program by the pseudospectral method, and then the solid line shows that the result calculated by Mathematica v.10.4.

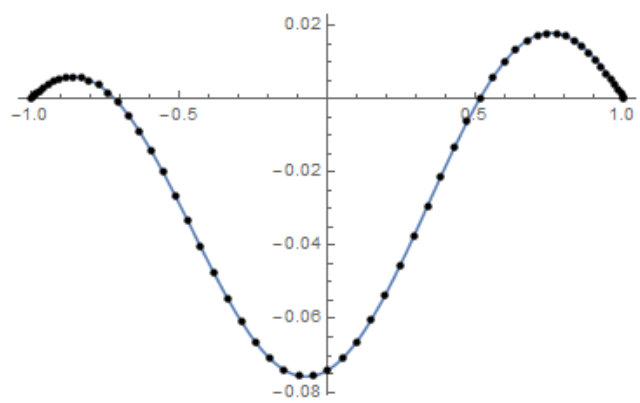

Figure 1: In case of The Hard Spring Duffing Oscillator with $\alpha=0.9, \beta=0.5, \gamma=0.7, \delta=0.65, \theta=4$.

Table 1: Comparison of numerical results and error with Mathematica calculations depend for Hard Spring Duffing Oscillator.

\begin{tabular}{lllll}
\hline$j$ & $x\left(t_{j}\right)$ & PS method & Mathematica 10.4 & Error \\
\hline 1 & 0.998795 & 0.000156882 & 0.000156814 & $6.84933 \times 10^{-8}$ \\
10 & 0.881921 & 0.126629 & 0.126628 & $7.41368 \times 10^{-8}$ \\
20 & 0.55557 & 0.00564902 & 0.00564893 & $9.18076 \times 10^{-8}$ \\
30 & 0.0980171 & -0.0662731 & -0.662732 & $1.12573 \times 10^{-7}$ \\
40 & -0.382683 & -0.0476281 & -0.0476282 & $1.23559 \times 10^{-7}$ \\
50 & -0.77301 & 0.00378497 & 0.00378492 & $5.64273 \times 10^{-8}$ \\
60 & -0.980785 & 0.00148411 & 0.0014861 & $6.74252 \times 10^{-8}$ \\
\hline
\end{tabular}

$b$. The Soft Spring Duffing Oscillator case

We gave the error $\varepsilon_{0}=10^{-8}$, the points $n=256$. And $\alpha=1, \beta=-0.7, \gamma=0.5, \delta=0.1, \theta=2 \pi$. Table 2 shows a comparison of numerical results and error with Mathematica calculations for Soft Spring Duffing Oscillator case. In the Fig. 2, the results were calculated base on the program by the pseudospectral method, and then the solid line shows that the result calculated by Mathematica v.10.4. 


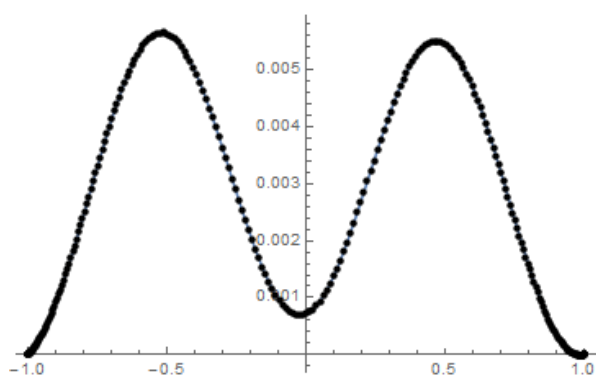

Figure 2: In case of The Soft Spring Duffing Oscillator with $\alpha=1, \beta=-0.7, \gamma=0.5, \delta=0.1, \theta=2 \pi$.

Table 2: Comparison of numerical results and error with Mathematica calculations for Soft Spring Duffing Oscillator.

\begin{tabular}{lllll}
\hline$j$ & $x\left(t_{j}\right)$ & PS method & Mathematica 10.4 & Error \\
\hline 1 & 0.999925 & $-1.15572 \times 10^{-7}$ & $-9.13587 \times 10^{-8}$ & $2.42141 \times 10^{-8}$ \\
50 & 0.817585 & 0.00127932 & 0.00127935 & $2.90011 \times 10^{-8}$ \\
100 & 0.33689 & 0.00470433 & 0.00470434 & $1.32052 \times 10^{-8}$ \\
150 & -0.266713 & 0.00308706 & 0.00308708 & $2.28701 \times 10^{-8}$ \\
200 & -0.77301 & 0.00291083 & 0.00291084 & $9.47306 \times 10^{-9}$ \\
250 & -0.99729 & $1.25929 \times 10^{-5}$ & $1.25938 \times 10^{-5}$ & $9.03146 \times 10^{-10}$ \\
\hline
\end{tabular}

c. The Inverted Duffing Oscillator case

We gave the error $\varepsilon_{0}=10^{-8}$, the points $n=64$. And $\alpha=2, \beta=0.7, \gamma=-1, \delta=0.5, \theta=2$. In the Table 3, we show competition the numerical results and error with Mathematica's calculations depend on the Inverted Duffing Oscillator. In the Fig. 3, the results were calculated base on the program by the pseudospectral method, and then the solid line shows that the result calculated by Mathematica v.10.4.

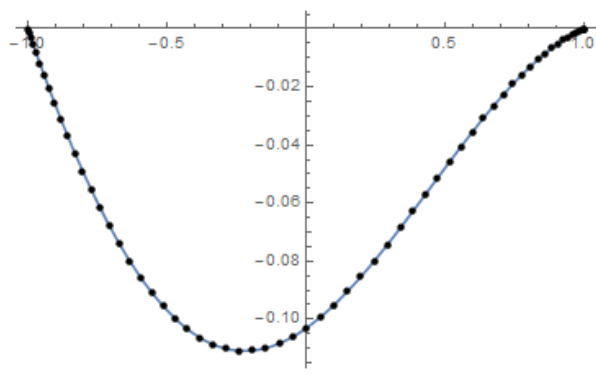

Figure 3: In case of The Inverted Duffing Oscillator with $\alpha=2, \beta=0.7, \gamma=-1, \delta=0.5, \theta=2$.

Table 3: Comparison of numerical results and error with Mathematica calculations for Inverted Duffing Oscillator.

\begin{tabular}{lllll}
\hline$j$ & $x\left(t_{j}\right)$ & PS method & Mathematica 10.4 & Error \\
\hline 1 & 0.998795 & $-4.87939 \times 10^{-5}$ & $-4.88112 \times 10^{-5}$ & $1.72549 \times 10^{-8}$ \\
10 & 0.881921 & -0.00668666 & -0.00668668 & $5.56609 \times 10^{-8}$ \\
20 & 0.55557 & -0.0405467 & -0.0405467 & $1.92109 \times 10^{-8}$ \\
30 & 0.0980171 & -0.0951851 & -0.0951851 & $3.53075 \times 10^{-9}$ \\
40 & -0.382683 & -0.106307 & -0.106307 & $5.53807 \times 10^{-9}$ \\
50 & -0.77301 & -0.0553363 & -0.0553363 & $2.6213 \times 10^{-9}$ \\
60 & -0.980785 & -0.00539904 & -0.00539904 & $4.57523 \times 10^{-9}$ \\
\hline
\end{tabular}

d. The Nonharmonic Duffing Oscillator case

We gave the error $\varepsilon_{0}=10^{-8}$, the points $n=128$. And $\alpha=5, \beta=0.9, \gamma=0, \delta=0.9, \theta=5$. Table 4 shows a comparison of numerical results and error with Mathematica calculations for Nonharmonic Duff- 
ing Oscillator case. In the Fig. 4, the results were calculated base on the program by the pseudospectral method, and then the solid line shows that the result calculated by Mathematica v.10.4.

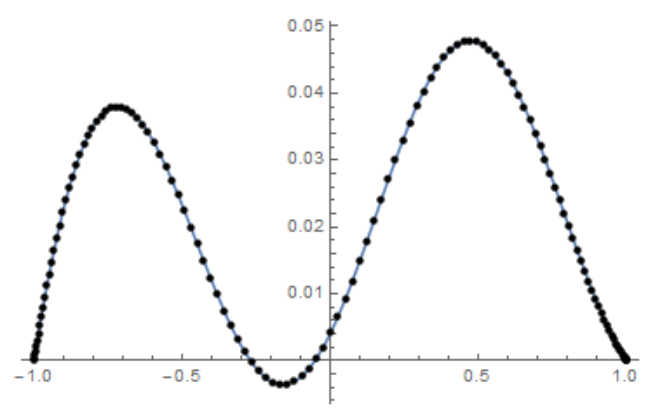

Figure 4: In case of The Nonharmonic Duffing Oscillator with $\alpha=5, \beta=0.9, \gamma=0, \delta=0.9, \theta=5$.

Table 4: Comparison of numerical results and error with Mathematica calculations depend for Nonharmonic Duffing Oscillator.

\begin{tabular}{lllll}
\hline $\mathfrak{j}$ & $x\left(\mathrm{t}_{\mathfrak{j}}\right)$ & PS method & Mathematica 10.4 & Error \\
\hline 1 & 0.999699 & $1.83279 \times 10^{-5}$ & $1.83161 \times 10^{-5}$ & $1.18298 \times 10^{-8}$ \\
20 & 0.881921 & 0.0105519 & 0.0105518 & $1.04707 \times 10^{-9}$ \\
40 & 0.55557 & 0.0455841 & 0.0455841 & $1.24329 \times 10^{-8}$ \\
60 & 0.0980171 & 0.0148238 & 0.0148237 & $1.94355 \times 10^{-8}$ \\
80 & -0.382683 & 0.00989231 & 0.0098923 & $1.12557 \times 10^{-8}$ \\
100 & -0.77301 & 0.0366845 & 0.0366845 & $1.39201 \times 10^{-8}$ \\
125 & -0.99729 & 0.000766572 & 0.000766565 & $7.55144 \times 10^{-9}$ \\
\hline
\end{tabular}

\section{Conclusion}

We used the pseudospectral method that used differential matrix for Chebyshev points to solve 4 special cases of the Duffing oscillator. The numerical results demonstrate the efficiency and the reliability method for solving this problem.

\section{Acknowledgment}

The publication was prepared with the support of the "RUDN University Program 5-100".

\section{References}

[1] M. A. Al-Jawary, S. G. Abd-Al-Razaq, Analytic and numerical solution for duffing equations, Int. J. Basic Appl. Sci., 5 (2016), 115-119. 1

[2] B. Bulbul, M. Sezer, Numerical Solution of Duffing Equation by Using an Improved Taylor Matrix Method, J. Appl. Math., 2013 (2013), 6 pages. 1

[3] W. S. Don, A. Solomonoff, Accuracy and speed in computing the Chebyshev collocation derivative, SIAM J. Sci. Comput., 16 (1995), 1253-1268. 2

[4] A. Elias-Ziga, O. Martnez-Romero, R. K. Crdoba-Daz, Approximate Solution for the Duffing-Harmonic Oscillator by the Enhanced Cubication Method, Math. Probl. Eng., 2012 (2012), 12 pages. 1

[5] A. O. El-Nady, M. M. A. Lashin, Approximate Solution of Nonlinear Duffing Oscillator Using Taylor Expansion, J. Mech. Engi. Auto., 6 (2016), 110-116. 1

[6] A. M. El-Naggar, G. M. Ismail, Analytical solution of strongly nonlinear Duffing Oscillators, Alex. Engi. Jour., 55 (2016), 1581-1585. 1

[7] R. H. Enns, G. C. McGuire, Nonlinear Physics with Mathematica for Scientists and Engineers, Birkhauser Basel, Boston, (2001). 1 
[8] M. Gorji-Bandpy, M. A. Azimi, M. M. Mostofi, Analytical methods to a generalized Duffing oscillator, Australian J. Basic Appl. Sci., 5 (2011), 788-796. 1

[9] M. A. Hosen, M. S. H. Chowdhury, M. Y. Ali, A. F. Ismail, An analytical approximation technique for the duffing oscillator based on the energy balance method, Ital. J. Pure Appl. Math., 37 (2017), 455-466. 1

[10] H.-Y. Lin, C.-C. Yen, K.-C. Jen, K. C. Jea, A Postverification Method for Solving Forced Duffing Oscillator Problems without Prescribed Periods, J. Appl. Math., 2014 (2014), 10 pages. 1

[11] J. C. Mason, D. C. Handscomb, Chebyshev Polynomials, Chapman \& Hall/CRC, Boca Raton, FL, (2003). 2, 2, 3

[12] S. Nourazar, A. Mirzabeigy, Approximate solution for nonlinear Duffing oscillator with damping effect using the modified differential transform method, Scientia Iranica, 20 (2013), 364-368. 1

[13] A. Pinelli, C. Benocci, M. Deville, A Chebyshev collocation algorithm for the solution of advection-diffusion equations, Comput. Methods Appl. Mech. Engrg., 116 (1997), 201-210. 1

[14] M. Razzaghi, G. Elnagar, Numerical solution of the controlled Duffing oscillator by the pseudospectral method, J. Comput. Appl. Math., 56 (1994), 253-261. 1

[15] A. Saadatmandi, F. Mashhadi-Fini, A pseudospectral method for nonlinear Duffing equation involving both integral and nonintegral forcing terms, Math. Methods Appl. Sci., 38 (2015), 1265-1272. 1

[16] A. H. Salas, J. E. Castillo H., Exact Solution to Duffing Equation and the Pendulum Equation, Appl. Math. Sci., 8 (2014), 8781-8789. 1 